\title{
Perfil de Ácidos Grasos y Evaluación de las Actividades Antioxidante y Antifúngica del Holotureo Isostichopus badionotus
}

\author{
Orlando J. Pastrana, Gilmar G. Santafé y Omar L. Torres \\ Universidad de Córdoba, Departamento de Química, Laboratorio Química de los productos naturales, \\ Carrera 6 No. 76-103, Montería, Córdoba-Colombia (e-mail: orlandojosepf@gmail.com, \\ gsantafe@correo.unicordoba.edu.co,omart2365@gmail.com
}

Recibido Sep. 2, 2015; Aceptado Nov. 9, 2015; Versión final Ene. 1, 2016, Publicado Jun. 2016

\begin{abstract}
Resumen
El extracto de diclorometano del holotureo Isostichopus badionotus, conocido vulgarmente como pepino de mar, mostró un total de 20 ácidos grasos con amplia diversidad estructural, los cuales fueron identificados por cromatografía de gases acoplada a espectrometría de masas. Los pepinos de mar han sido introducidos a la dieta humana, principalmente en los países asiáticos y europeos, debido a su contenido de nutrientes y su relativo bajo costo. De ahí surge el interés en estudiar sus propiedades nutritivas. Se determinó el contenido total de fenoles y las actividades antioxidante y antifúngica de los extractos metanólico, acuoso y de diclorometano del organismo, encontrándose un bajo contenido de fenoles $(<6$ $\mathrm{mg} \mathrm{EAG/g})$, baja actividad antioxidante por los métodos ABTS y DPPH $\left(\mathrm{Cl}_{50}>100 \mu \mathrm{g} / \mathrm{mL}\right)$ y bajo potencial de reducción férrica $(<3 \mathrm{mg} E A A / g)$. También se determinó que ninguno de los extractos a las concentraciones evaluadas mostró actividad inhibitoria frente a los hongos fitopatógenos estudiados.
\end{abstract}

Palabras clave: ácidos grasos; actividad antioxidante; actividad antifúngica; Holotúreos; Isostichopus badionotus; hongos fitopatógenos

\section{Fatty Acids Profile and Evaluation of the Antioxidant and Antifungal Activities of Holothuria Isostichopus badionotus}

\begin{abstract}
The dichloromethane extract from the holothuria Isostichopus badionotus, commonly known as sea cucumbers, showed a total of 20 fatty acids with a large structural diversity, which were identified by gas chromatography coupled to mass spectrometry. Sea cucumbers have been incorporated into human diet mainly in Asia and some European countries due to their nutrient content and their relatively low cost. These facts have attracted the interest in studying the nutrient properties of these marine animals The total phenolic content and antioxidant and antifungal activities of methanol, aqueous and dichloromethane extracts of the organism were determined finding a low phenolic content $(<6 \mathrm{mg} \mathrm{EAG/g})$, low antioxidant activity by ABTS and DPPH methods $\left(\mathrm{IC}_{50}>100 \mu \mathrm{g} / \mathrm{mL}\right.$ ) and low ferric reduction potential $(<3 \mathrm{mg} \mathrm{EAA} / \mathrm{g})$. It was also determined that none of the extracts at the evaluated concentrations showed inhibitory activity against any of the phytopathogenic fungi studied.
\end{abstract}

Keywords: fatty acids, antioxidant activity; antifungal activity; holothurians; Isostichopus badionotus; phytopathogenic fungi 


\section{INTRODUCCIÓN}

Los holotureos comúnmente son conocidos como pepinos de mar debido a su apariencia similar a sus homónimos de las plantas, en el mundo existen alrededor de 1500 especies, de las cuales aproximadamente 50 son comestibles e inclusive cultivables, entre ellas se encuentra Isostichopus badionotus (Zamorano et al., 2005). Los pepinos de mar han sido introducidos a la dieta humana, principalmente en los países asiáticos y europeos, debido a su contenido de nutrientes y su relativo bajo costo si se compara con otros organismos marinos que aportan básicamente la misma variedad de nutrientes a los humanos (Purcell et al., 2012). Entre los principales aportes nutricionales de los pepinos de mar están los ácidos grasos poliinsaturados destacándose compuestos como los ácidos araquidónico, eicosapentanoico y docosahexanoico, cuya biosíntesis se encuentra asociada específicamente a los holotureos (Saito et al., 2014). Este tipo de ácidos grasos son considerados de beneficio para la salud humana, su consumo contribuye a reducir los riesgos de sufrir enfermedades cardiovasculares, también son conocidos por tener actividad anticancerígena, efectos antiinflamatorios, previenen la osteoporosis y los disturbios neurológicos actuando como agentes que tienen la capacidad de reducir las conductas depresivas en las personas (Macedo y Vélez, 2015; Cawood et al., 2010).

La dieta de los holotureos se fundamenta principalmente en el consumo de algas y fitoplancton, este factor origina la gran variedad de ácidos grasos presentes (Gu et al., 2012), en los cuales las cadenas carbonadas insaturadas se encuentran directamente relacionadas con un potencial antioxidante moderado basado en la capacidad de estabilizar los radicales libres $(R L)$ y algunas especies reactivas de oxígeno (ERO) (Sanhueza et al., 2002). Si bien, en los procesos químicos o biológicos normales se producen radicales libres o especies reactivas de oxígeno, en los organismos vivos existen una gran cantidad de eventos que contribuyen a desplazar el equilibrio entre las concentraciones de oxidantes y antioxidantes (Zapata et al., 2013), este fenómeno es conocido como estrés oxidativo y genera daños significativos en el organismo, afectando numerosos procesos en el aparato cardiovascular (aterosclerosis, cardiopatía alcohólica), sistema neurológico (enfermedad de Parkinson, Alzheimer, traumatismos craneales), aparato ocular (catarata, fibroplasia), aparato respiratorio (cáncer de pulmón), riñón (nefrotoxicidad por metales) y artritis reumatoidea (Granados et al., 2013; Murillo et al., 2007); esta sumatoria de eventos prácticamente ha obligado a las personas a incluir en la dieta alimentos con potencial antioxidante para restablecer el equilibrio en el organismo (Contreras et al., 2010). Para los pepinos de mar, parte de su potencial antioxidante está entonces asociado a su contenido de ácidos grasos insaturados, por supuesto, la presencia de otro tipo de compuestos químicos como por ejemplo derivados fenólicos o flavonoides también contribuyen al desarrollo de su capacidad antioxidante (Althunibat et al., 2009; Mamelona et al., 2007).

Los pepinos de mar son organismos con un sistema inmune bastante desarrollado (Arizza et al., 2013; Zhao et al., 2012), esta capacidad biológica les ha permitido biosintetizar o almacenar compuestos químicos que han demostrado poseer gran potencial antifúngico frente a varios géneros de hongos patógenos y fitopatógenos (Santafé et al., 2014; Chen et al., 2012; Wang et al., 2012, Han et al., 2009, Ismail et al., 2008, Kumar et al., 2007), posiblemente como un mecanismo de adaptación a los diferentes hábitats en los que se desarrollan (Pastrana et al., 2015). Este potencial de los holotureos para producir compuestos antifúngicos ha despertado el interés de la comunidad científica por encontrar sustancias con este tipo de actividad biológica, debido entre otros aspectos, a la resistencia que han adquirido los hongos a muchas de las sustancias utilizadas para su tratamiento (Sánchez et al, 2015; Sanabria et al., 2014). Los hongos patógenos ocasionan daños considerables en la salud humana pudiendo llegar a ser mortales sin el tratamiento adecuado (Sánchez y Almaguer, 2014), por su parte los hongos fitopatógenos destruyen toneladas de cultivos anualmente en todo el mundo, este comportamiento está directamente relacionado con la capacidad que tiene los hongos para atacar a la planta en todas las etapas de su desarrollo (González et al., 2006).

En la presente investigación se describe el perfil de los ácidos grasos del pepino de mar Isostichopus badionotus, el cual es uno de los holotureos más valorado y consumido como alimento por los humanos. También, se muestran los resultados de los ensayos de las actividades antioxidante y antifúngica realizados a los extractos obtenidos de este organismo, buscando la posibilidad de encontrar mezclas de sustancias 0 compuestos puros que puedan llegar a tener aplicación en el tratamiento del estrés oxidativo o de los problemas ocasionados por hongos.

\section{MATERIALES Y MÉTODOS}

Material biológico: Los especímenes del pepino de mar I. badionotus se recolectaron en la Bahía de Cispatá (Latitud: $9.38^{\circ} \mathrm{N}$, Longitud: $75.79^{\circ} \mathrm{O}$ ), en el Caribe Colombiano (Quirós y Arias, 2013). El material se sometió a congelación hasta posterior tratamiento. Los especímenes recolectados se ubicaron taxonómicamente y un ejemplar de colección de cada especie se encuentra en el laboratorio de Química de los Productos Naturales de la Universidad de Córdoba, Colombia, con el código PNM 028. 
Obtención de los extractos: El material recolectado de I. badionotus se cortó en pedazos y se sometió a percolación en metanol ( $\mathrm{MeOH}$ ) durante cinco días (3 repeticiones), se filtró y se concentró a presión reducida obteniendo el extracto metanólico, posteriormente se sometió a fraccionamiento por reparto empleando diclorometano (DCM) y agua, la fase orgánica se separó y se concentró a presión reducida hasta obtener el extracto de DCM, mientras que la fase acuosa se sometió a liofilización obteniendo el extracto acuoso (Pastrana et al., 2015).

Obtención de la fracción de ácidos grasos: El extracto de DCM se sometió a cromatografía en columna (CC) sobre sílica gel usando sistemas de elución desde Bencina:AcOEt (7:1) hasta Metanol (MeOH), las subfracciones obtenidas se monitorearon por cromatografía de capa delgada (CCD). Como reveladores se utilizaron luz ultravioleta, vapores de yodo, ácido fosfomolíbdico y vainillina. Las subfracciones con Rf similar al ácido oleico se reunieron, se concentraron y se sometieron a esterificación para su posterior a análisis por cromatografía de gases acoplado a espectrometría de masas (CG-EM) (Santafé et al., 2009).

Obtención de ésteres metílicos de ácidos grasos: Los ésteres metílicos de ácidos grasos se obtuvieron empleando una esterificación en medio básico, para lo cual se tomaron $190 \mathrm{mg}$ de la fracción de ácidos grasos se colocaron a reflujo con $4 \mathrm{~mL}$ de solución $2 \mathrm{~N}$ de hidróxido de potasio $(\mathrm{KOH})$ en metanol a $80^{\circ} \mathrm{C}$ por $3 \mathrm{~h}$ con agitación magnética. La mezcla se enfrió y se monitoreó la saponificación por CCD usando Bencina:Acetato de etilo $(9: 1 \mathrm{v} / \mathrm{v})$ y como reveladores vapores de yodo y ácido fosfomolíbdico. Posteriormente el producto obtenido se combinó con $3 \mathrm{~mL}$ de solución al $20 \%$ de trifluoruro de boro metanólico $\left(\mathrm{BF}_{3} / \mathrm{MeOH}\right)$ y se sometió a reflujo por $3 \mathrm{~h}$ y $80^{\circ} \mathrm{C}$. La esterificación se verificó por CCD usando el mismo sistema de elución anterior. La mezcla de reacción se extrajo con DCM y se lavó con solución saturada de cloruro de sodio, y la fase orgánica se lavó con sulfato de sodio anhidro, se filtró y se concentró a $37^{\circ} \mathrm{C}$ por destilación a presión reducida (Rodríguez, 2005). Las impurezas se eliminaron empleando CC sobre sílica gel con el sistema de elución antes mencionado y posteriormente la mezcla de ésteres metílicos purificados se analizó por CG-EM.

Condiciones cromatográficas: Para la obtención del cromatograma y los espectros masas de los ésteres metílicos de los ácidos grasos se empleó un cromatógrafo de gases Hewlett Packard $6890 \mathrm{~N}$, con columna capilar Modelo Agilent 122-1032 DB-1 de $0.25 \mathrm{~mm}$ de diámetro externo, $30.6 \mathrm{~m}$ de largo y $0.25 \mu \mathrm{m}$ de diámetro interno, acoplado a un espectrómetro de masas Hewlett Packard 6890, con detector selectivo de masas modelo $5973 \mathrm{~N}$ y fuente de ionización de $70 \mathrm{eV}$. Para el cromatografo de gases se empleó el método de inyección Splitless con un volumen de inyección de $1 \mu \mathrm{L}$, se empleó helio como gas carrier y una rampa de calentamiento de $5^{\circ} \mathrm{C} / \mathrm{min}$ con temperatura inicial de $100^{\circ} \mathrm{C}$ y temperatura final de $325^{\circ} \mathrm{C}$, flujo nominal de $1 \mathrm{~mL} / \mathrm{min}$ y un tiempo total de análisis de $60 \mathrm{~min}$. Para el espectrómetro de masas se empleó el modo de adquisición scan con parámetros de $\mathrm{m} / \mathrm{z}$ de 30.0 a 800.0 y una temperatura del detector entre 150 y $200^{\circ} \mathrm{C}$.

Identificación de los ésteres metílicos: Los ésteres metílicos de los ácidos grasos se identificaron por análisis de sus patrones de fragmentación obtenidos por EM y comparación con bases de datos y bibliografía especializada. Los porcentajes de abundancia de los ésteres metílicos se determinaron por relación directa de pico de cada éster con el área total del cromatograma.

Determinación del contenido total de fenoles: El contenido total de fenoles se determinó mediante el método espectrofotométrico de Folin-Ciocalteu (Benzie y Strain, 1996). Se preparó una disolución patrón de ácido gálico de $100 \mu \mathrm{g} / \mathrm{mL}$ ( $5 \mathrm{mg}$ de ácido gálico en $50 \mathrm{~mL}$ agua grado HPLC), una dilución 1:10 con agua grado HPLC, una disolución de carbonato de sodio al $20 \%$ y una disolución $1 \mathrm{~N}$ del reactivo de Folin Ciocalteau, por medio de una dilución 1:2 del reactivo comercial $(2 \mathrm{~N})$; el reactivo se protegió de la luz y se colocó en refrigeración hasta su uso. A partir de la disolución patrón de ácido gálico, en viales protegidos de la luz se hicieron las diluciones de 1,2, 3, 4 y $5 \mu \mathrm{g} / \mathrm{mL}$ para la curva de calibración. Esto se realizó tomando respectivamente $20,40,60,80$ y $100 \mu \mathrm{L}$ de la disolución patrón de ácido gálico de $100 \mu \mathrm{g} / \mathrm{mL}$, luego se adicionó a cada uno $250 \mu \mathrm{L}$ de reactivo de Folin Ciocalteau $1 \mathrm{~N}$. La mezcla se agitó durante 5 min, posteriormente se adicionaron $1.250 \mu \mathrm{L}$ de la disolución de carbonato de sodio al $20 \%$, luego se llevó a un volumen final de $2000 \mu \mathrm{L}$ con agua grado HPLC y se dejó reposar por $90 \mathrm{~min}$. Se analizó blanco que contenía todos los componentes excepto el reactivo de Folin Ciocalteau (García et al., 2011), finalmente se leyó la absorbancia a $760 \mathrm{~nm}$. El contenido de fenoles de cada extracto se determinó de forma similar al procedimiento utilizado para el ácido gálico y su valor es expresado como $\mathrm{mg}$ de equivalentes de ácido gálico/g de peso seco de muestra.

Potencial de Reducción Férrica: Se preparó una solución madre de TPTZ (2,4,6-tri(2-piridil)-1,3,5-triazina) (25 $\mathrm{mL}$ de solución buffer de acetato $300 \mathrm{mM}$; pH 3.6 con $2.5 \mathrm{~mL}$ de solución de TPTZ $10 \mathrm{mM}(0,083 \mathrm{~g}$ de TPTZ aforados a $10 \mathrm{~mL}$ ) en $\mathrm{HCl} 40 \mathrm{mM}\left(1.7 \mathrm{~mL}\right.$ de $\mathrm{HCl}$ aforados en $500 \mathrm{~mL}$ de $\mathrm{H}_{2} \mathrm{O}$ ) y $2.5 \mathrm{~mL}$ de $\mathrm{FeCl}_{3}{ }^{*} 6 \mathrm{H}_{2} \mathrm{O} 20$ $\mathrm{mM}\left(0.540 \mathrm{~g}\right.$ de $\mathrm{FeCl}_{3}{ }^{*} 6 \mathrm{H}_{2} \mathrm{O}$ aforados a $100 \mathrm{~mL}$ de $\left.\mathrm{H}_{2} \mathrm{O}\right)$ ). Para la evaluación del potencial de reducción se 
mezclaron $1800 \mu \mathrm{L}$ de solución madre de TPTZ previamente preparada, con $100 \mu \mathrm{L}$ de muestra y $100 \mu \mathrm{L}$ de agua destilada. Luego se incubó la mezcla a temperatura ambiente durante 60 min y se midió la absorbancia a una longitud de onda de $593 \mathrm{~nm}$. Para cada muestra se tuvo en cuenta la lectura de la absorbancia del blanco que no contenía TPTZ y se utilizó ácido ascórbico como patrón de referencia para la elaboración de la curva de calibrado (Rojano et al. 2008).

Actividad Antifúngica: Esta se evaluó frente a los hongos fitopatógenos Sclerotium sp., Rhizoctonia sp. y Fusarium sp. empleando el método de difusión en discos sobre agar. Los hongos se inocularon en cajas Petri empleando caldo PDA (34 g/L) como medio de cultivo y se incubaron en un período de cinco días a una temperatura de $20^{\circ} \mathrm{C}$. A partir de este cultivo con ayuda de un sacabocados de $8 \mathrm{~mm}$ de diámetro se tomó un disco de hongo y se inoculó en el centro de la caja de Petri. Sobre la superficie del agar se depositaron discos Whatman de $5 \mathrm{~mm}$ de diámetro previamente impregnados con soluciones de los extractos a concentraciones de 50,100, 500 y $2000 \mu \mathrm{g} / \mathrm{mL}$. Como control negativo se emplearon discos impregnados con disolvente (DMSO) y como control positivo discos impregnados con soluciones de Propical $\AA^{\circledR}$ a $500 \mu \mathrm{g} / \mathrm{mL}$, todas las cajas se incubaron en un período de cinco días a una temperatura de $20^{\circ} \mathrm{C}$ y el potencial antifúngico se determinó por medición de los halos de inhibición (Pastrana et al., 2015).

\section{RESULTADOS Y DISCUSIÓN}

El análisis de los espectros de masas de los ésteres metílicos de ácidos grasos obtenidos del pepino de mar I. badionotus reveló la presencia de 20 compuestos, los cuales se muestran en la tabla 1 .

Tabla 1: Ésteres metílicos de ácidos grasos identificados del pepino de mar I. badionotus

\begin{tabular}{|c|c|c|}
\hline $\begin{array}{c}\text { Tiempo de } \\
\text { Retención (min) }\end{array}$ & Porcentaje (\%) & Compuesto Identificado \\
\hline 16.94 & 1.19 & Éster metílico del ácido 12-tetradecenoico \\
\hline 18.05 & 2.37 & Éster metílico del ácido tetradecanoico \\
\hline 18.38 & 3.50 & Éster metílico del ácido 13-metiltetradecanoico \\
\hline 18.98 & 1.56 & Éster metílico del ácido 12-metiltetradecanoico \\
\hline 19.34 & 2.81 & Éster metílico del ácido pentadecanoico \\
\hline 20.53 & 2.88 & Éster metílico del ácido 14-metilpentadecanoico \\
\hline 20.70 & 13.95 & Éster metílico del ácido 14-hexadecenoico \\
\hline 20.96 & 1.62 & Éster metílico del ácido 9-hexadecenoico \\
\hline 21.68 & 18.79 & Éster metílico del ácido hexadecanoico \\
\hline 22.54 & 2.28 & Éster metílico del ácido 15-metilhexadecanoico \\
\hline 23.12 & 2.97 & Éster metílico del ácido 15-heptadecenoico \\
\hline 24.63 & 2.61 & Éster metílico del ácido heptadecanoico \\
\hline 24.86 & 0.93 & Éster metílico del ácido 12,16-Octadecadienoico \\
\hline 25.19 & 4.48 & Éster metílico del ácido 16-Octadecenoico \\
\hline 25.77 & 6.49 & Éster metílico del ácido Octadecanoico \\
\hline 26.78 & 0.65 & Éster metílico del ácido 17-Nonadecenoico \\
\hline 27.83 & 1.00 & Éster metílico del ácido Nonadecanoico \\
\hline 28.48 & 9.20 & Éster metílico del ácido $12,14,16,18$-Eicosatetraenoico \\
\hline 30.40 & 2.11 & Éster metílico del ácido 18-Eicosenoico \\
\hline 31.20 & 0.75 & Éster metílico del ácido Eicosanoico \\
\hline
\end{tabular}

Realizando en análisis comparativo del perfil de ácidos grasos que fueron identificados a partir del pepino de mar I. badionotus, agrupados a partir de sus características estructurales, se observó que los ácidos grasos de cadena par y los de cadena impar que se encuentran presentes en este organismo no son proporcionales, mostrando una prevalencia hacia los ácidos grasos de cadena par con un porcentaje de $67.73 \%$, frente a $14.41 \%$ para los de cadena impar, asimismo se observó una diferencia bastante marcada en las proporciones de los ácidos grasos de cadena lineal (71.92\%), sobre los de cadena ramificada (10.22\%). En este organismo no se identificaron ácidos grasos con cadenas laterales superiores a 24 carbonos, y la proporción en la que se encuentran los ácidos grasos con cadenas laterales entre 20 y 24 carbonos es mucho menor (12.06\%) que la de ácidos grasos con cadenas laterales menores a 20 carbonos $(70.08 \%)$. 
En el pepino de mar I. badionotus, fue encontrada una mayor proporción de ácidos grasos saturados AGS (45.04 \%) frente a los ácidos grasos monoinsaturados AGMI (26.97\%). Por su parte, se encontró un bajo contenido contenido de ácidos grasos poliinsaturados AGPI con solo el $9.20 \%$ del contenido total de ácidos grasos, este valor se encuentra por debajo de los porcentajes reportados para pepinos de mar que oscilan entre 20 y 35\% (Drazen et al., 2008; Fredalina et al., 2000; Carballeira et al., 1996), dado que estos organismos se alimentan principalmente las algas y fitoplancton, los cuales son grandes productores de AGPI (Gu et al., 2012). Los ácidos grasos con mayor porcentaje de abundancia encontrados a partir del análisis por cromatografía de gases fueron: el ácido hexadecanoico (18.79\%), el ácido 14-hexadecenoico (13.95\%), el ácido $12,14,16,18$-Eicosatetraenoico $(9.20 \%)$, el ácido Octadecanoico (6.49\%) y el ácido 16-Octadecenoico (4.48\%). No se encontraron los ácidos araquidónico, eicosapentaenoico y docosahexaenoico, los cuales se encuentran bastante asociados a equinodermos (Saito et al., 2014), sin embargo, se encontró AGMI y AGS de la serie $\mathrm{C}_{20}$, como los ácidos eicosenoico y eicosanoico, para los cuales se ha establecido que su principal precursor es el ácido araquidónico, esto sugiere que posiblemente este organismo modifica estructuralmente los AGPI que adquiere en la dieta, convirtiéndolos en AGS y AGMI (Miles et al., 2012). La ausencia de ácidos grasos poliinsaturados junto con la de otros ácidos grasos de las series $\omega-3$ y $\omega-6$, hacen que $I$. badionotus tenga un muy bajo interés nutricional para la dieta humana desde el punto de vista del aporte de ácidos grasos benéficos para la salud (Macedo y Vélez, 2015; Gómez et al., 2011; Cawood et al., 2010), sin embargo, este organismo es uno de los más apetecidos y comercializados en los mercados de muchos países europeos y asiáticos (Purcell et al., 2012).

El contenido total de fenoles (Tabla 2) muestran valores inferiores a $5 \mathrm{mg} \mathrm{EAG/g} \mathrm{de} \mathrm{extracto,} \mathrm{lo} \mathrm{que} \mathrm{concuerda}$ con reportes previos en equinodermos (entre 1.5 y $7.0 \mathrm{mg} \mathrm{EAG/g} \mathrm{de} \mathrm{extracto)} \mathrm{(Mamelona} \mathrm{et} \mathrm{al,} \mathrm{2007),} \mathrm{aunque}$ estos pueden ser mayores. El potencial antioxidante de un extracto se encuentra íntimamente ligado a compuestos fenólicos (Palomino et al., 2009), sin embargo no se ha logrado establecer con certeza una relación directa entre el contenido de compuestos fenólicos y el potencial antioxidante de extractos, debido a que estos son matrices muy complejas sobre las cuales se ejercen efectos sinérgicos o antagónicos que pueden variar considerablemente el potencial antioxidante (García et al., 2011). Los equinodermos no son capaces de producir compuestos fenólicos, ya que estos se encuentran exclusivamente asociados al metabolismo secundario de las plantas (Kuskoski et al., 2005), inclusive estos compuestos son considerados tóxicos para los invertebrados marinos a concentraciones superiores a $1 \mathrm{mg} E A G / g$ de organismo, sin embargo, la mayoría de los invertebrados marinos, principalmente los equinodermos, tienen como principal característica la capacidad asimilar y bioacumular estos compuestos a concentraciones moderadas, ya que los obtienen de la alimentación diaria que se fundamenta principalmente en algas, las cuales son grandes productoras de éstos compuestos (Althunibat et al. 2009).

Tabla 2: Contenido total de fenoles y actividad antioxidante de los extractos del pepino de mar I. badionotus.

\begin{tabular}{|l|c|c|}
\hline \multicolumn{1}{|c|}{ Extractos } & $\begin{array}{c}\text { Contenido Total de } \\
\text { Fenoles }\end{array}$ & $\begin{array}{c}\text { Potencial de } \\
\text { Reducción Férrica }\end{array}$ \\
\hline Metanólico & $4.77 \mathrm{mg} \mathrm{EAG/g}$ & $1.97 \mathrm{mg} \mathrm{EAA} / \mathrm{g}$ \\
\hline Diclorometano & $1.34 \mathrm{mg} \mathrm{EAG/g}$ & $1.04 \mathrm{mg} \mathrm{EAA} / \mathrm{g}$ \\
\hline Acuoso & $5.49 \mathrm{mg} \mathrm{EAG/g}$ & $2.99 \mathrm{mg} \mathrm{EAA} / \mathrm{g}$ \\
\hline
\end{tabular}

Se encontró un bajo potencial de reducción férrica para los extractos de I. badionotus con un valor máximo para el extracto acuoso de $2.99 \mathrm{mg} \mathrm{EAA} / \mathrm{g}$ de extracto. Lo anterior concuerda con el bajo contenido de compuestos fenólicos encontrado en los diferentes extractos, debido a que el potencial de reducción está ligado a la capacidad de donar electrones a los compuestos presentes en el extracto, la cual es una de las principales características de los compuestos fenólicos polihidroxilados (Zhou et al. 2011).

La evaluación de la actividad antifúngica (Tabla 3) mostró que los extractos son inactivos contra todos hongos fitopatógenos a las condiciones empleadas. El control positivo (Propical®) mostró halos de inhibición de 23 y $18 \mathrm{~mm}$ para Fusarium sp. y Rhizoctonia sp., respectivamente. Lo anterior posiblemente puede resultar de la ausencia de compuestos antimicrobianos en los extractos (Calvo et al., 2009), o de efectos antagónicos entre los constituyentes de los extractos (Flórez et al., 2007). La ausencia de potencial antifúngico de los extractos de $I$. badionotus resulta ser un hecho interesante, ya que varios estudios han demostrado el potencial antifúngico de los tirterpenos producidos por los pepinos de mar (Santafé et al., 2014; Chen et al., 2012; Wang et al., 2012, Han et al., 2009, Ismail et al., 2008, Kumar et al., 2007), por lo que estudios posteriores deben ser realizados para establecer la presencia/ausencia de esta clase de compuestos en los extractos de $I$. badionotus. 


\section{CONCLUSIONES}

El estudio realizado evidenció la prevalencia hacia la producción de ácidos grasos saturados por parte del holotureo o pepino de mar Isostichopus badionotus, así como los bajos porcentajes de ácidos grasos poliinsaturados y la ausencia de ácidos grasos de la serie $\omega-6$, estas características disminuyen el aporte nutricional de este organismo en la dieta humana. No obstante, el conocimiento detallado del perfil de ácidos grasos de I. badionotus contribuye a ampliar el conocimiento químico en torno al estudio de los holotureos. Los extractos estudiados mostraron un bajo contenido total de fenoles, resultado que está de acuerdo con el bajo potencial reductor encontrado. De otro parte, también se evidenció inactividad de los extractos frente a las cepas de los hongos fitopatógenos a las condiciones evaluadas, debido posiblemente a la ausencia de compuestos con actividad antifúngica en este organismo.

\section{AGRADECIMIENTOS}

Los autores expresan su agradecimiento a la Oficina de Investigación de la Universidad de Córdoba, Colombia, por la financiación de este trabajo.

\section{REFERENCIAS}

Althunibat, O.Y. y otros 5 autores. In Vitro Antioxidant and Antiproliferative Activities of Three Malaysian Sea Cucumber Species. European Journal of Scientific Research: 37, 376-387 (2009)

Arizza, V. y otros 5 autores. Gender differences in the immune system activities of sea urchin Paracentrotus lividus. Comparative Biochemistry and Physiology, Part A: 164, 447-455 (2013)

Benzie, I. y Strain, J., The Ferric Reducing Ability of Plasma (FRAP) as a Measure of "Antioxidant Power": The FRAP Assay. Analytical Biochemistry: 239, 70-76 (1996)

Calvo, J. y Martínez, L., Mecanismos de acción de los antimicrobianos. Enfermedades Infecciosas y Microbiología Clínica: 27, 44-52 (2009)

Carballeira, N.; Cruz, C. y Sostre, A., Identification of the Novel 7-Methyl-6-octadecenoic Acid in Holothuria mexicana. Journal of Natural Products: 59, 1076-1078 (1996)

Cawood, A. y otros 13 autores. Eicosapentaenoic acid (EPA) from highly concentrated n-3 fatty acid ethyl esters is incorporated into advanced atherosclerotic plaques and higher plaque EPA is associated with decreased plaque inflammation and increased stability. Atherosclerosis: 212, 252-259 (2010)

Chen, S. y otros 6 autores. Sequence determination and anticoagulant and antithrombotic activities of a novel sulfated fucan isolated from the sea cucumber Isostichopus badionotus. Biochimica et Biophysica Acta: 1820, 989-1000 (2012)

Contreras, J.; Calderon, L.; Guerra, E. y Garcia, B., Antioxidant capacity, phenolic content and vitamin C in pulp, peel and seed from 24 exotic fruits from Colombia. Food Research International: 44 (7), 2047-2053 (2010)

Drazen, J.; Phleger, C. y Nichols P., Lipid, sterols and fatty acid composition of abyssal holothurians and ophiuroids from the North-East Pacific Ocean: Food web implications. Comparative Biochemistry and Physiology Part B: 151, 79-87 (2008)

Flórez, M., D’Armas, H. y Herrera H., Identificación de algunos constituyentes químicos de las hojas de Chromolaena laevigata mediante cromatografía de gas-espectrometría de masas. Ciencia: 15, 1-12 (2007)

Fredalina, B.D. y otros 7 autores. Fatty acid compositions in local sea cucumber, Stichopus chloronotus, for wound healing. General Pharmacology: 33, 337-340 (2000)

García, J.R. y otros 7 autores. Cuantificación de polifenoles y capacidad antioxidante en duraznos comercializados en la ciudad de Juárez, México. Tecnociencia: 5(2), 67-75 (2011)

Gómez C.; Bermejo, L. y Kohen, V., Importance of a balanced omega 6/ omega 3 ratio for the maintenance of health. Nutrición Hospitalaria: 26(2), 323-329 (2011)

González, V.; Portal, M. y Rubio, V., Review. Biology and Systematics of the form genus Rhizoctonia. Spanish Journal of Agricultural Research: 4(1), 55-79 (2006) 
Granados, C.; Yáñez, X. y Acevedo, D., Evaluación de la Actividad Antioxidante del Aceite Esencial Foliar de Myrcianthes leucoxyla de Norte de Santander (Colombia). Información Tecnológica: 25(3), 11-16 (2014)

$\mathrm{Gu}, \mathrm{M}$. y otros 5 autores. Effects of dietary b-glucan, mannan oligosaccharide and their combinations on growth performance, immunity and resistance against Vibrio splendidus of sea cucumber, Apostichopus japonicus. Fish \& Shellfish Immunology: 31, 303-309 (2012)

Han, H. y otros 5 autores. Antifungal active triterpene glycosides from sea cucumber Holothuria scabra. Acta Pharmaceutica Sinica: 44(6), 620-624 (2009)

Ismail, $\mathrm{H}$. y otros 7 autores. Antifungal activity of aqueous and methanolic extracts from the Mediterranean sea cucumber, Holothuria polii. Journal of Mycologie Médicale: 18, 23-26 (2008)

Kumar, R.; Kumar, A.; Kumar, P. y Lakshmi, V. Antifungal activity in triterpene glycosides from the sea cucumber Actinopyga lecanora. Bioorganic \& Medicinal Chemistry Letters: 17, 4387-4391 (2007)

Kuskoski. E.; Asuro, A.; Troncoso, A.; Fett, R.; Mancini-Filho, J., Aplicación de diversos métodos químicos para determinar actividad antioxidante en pulpa de frutos. Ciência e Tecnologia de Alimentos: 25(4), 726-732 (2005)

Macedo, R. y Vélez, J., Propiedades Fisicoquímicas y de Flujo de un Yogur Asentado Enriquecido con Microcápsulas que Contienen Ácidos Grasos Omega 3. Información Tecnológica: 26(5), 87-96 (2015)

Mamelona, J. y otros 5 autores. Quantification of phenolic contents and antioxidant capacity of Atlantic sea cucumber, Cucumaria frondosa. Food Chemistry: 104, 1040-1047 (2007)

Miles, E. y Calder, P., Influence of marine $n-3$ polyunsaturated fatty acids on immune function and a systematic review of their effects on clinical outcomes in rheumatoid arthritis. British Journal of Nutrition: 107, 171-184 (2012)

Murillo, E.; Lombo, O.; Tique, M. y Méndez, J., Potencial Antioxidante de Bauhinia Kalbreyeri Harms (FABACEAE). Información Tecnológica: 18(6), 65-74 (2007)

Palomino, L.R.; García, C.M.; Gil, J.H.; Rojano, B.A. y Durango, D.L., Determinación del contenido de fenoles y evaluación de la actividad antioxidante de propóleos recolectados en el departamento de Antioquia (Colombia). Vitae: 16(3), 388-395 (2009)

Pastrana, O.; Santafé, G. y Angulo, A., Actividad antibacteriana y antifúngica de la estrella de mar Oreaster reticulatus (Valvatida: Oreasteridae) y de los erizos de mar Mellita quinquiesperforata (Clypeasteroida: Mellitidae) y Diadema antillarum (Diadematoida: Diadematidae) del Caribe Colombiano. Revista Biología Tropical: 63(2), 329-337 (2015)

Purcell, S.W.; Hair, C.A. y Mills, D.J., Sea cucumber culture, farming and sea ranching in the tropics: Progress, problems and opportunities. Aquaculture: 368-369, 68-81 (2012)

Quirós, J.A.; Arias, J.E., Taxocenosis de Moluscos y Crustáceos en raíces de Rhizophora mangle (Rhizophoraceae) en la Bahía de Cispatá, Córdoba, Colombia. Acta biológica Colombiana: 18(2), 329-340 (2013)

Rodríguez, M., Estudio comparativo de la composición de ácidos grasos del aceite de semilla de plantas de la Amazonia colombiana. Momentos de Ciencia: 2(2), 75-81 (2005)

Rojano B.A. y otros 5 autores. Actividad antioxidante del isoespintanol en diferentes medios. Vitae: 15(1), 173-181 (2008)

Saito, H. y Aono, H., Characteristics of lipid and fatty acid of marine gastropod Turbo cornutus: High levels of arachidonic and n-3 docosapentaenoic acid. Food Chemistry: 145, 135-144 (2014)

Sanabría, R. y otros 6 autores. Perfil de susceptibilidad a anti fúngicos de aislados de Candida spp por el método de microdilución. Nuevos puntos de cortes para fluconazol. Memorias del Instituto de Investigaciones en Ciencias de la Salud: 12(1), 33-40 (2014)

Sánchez, G.; Vargas, A. y Jiménez, P., Evaluación de la actividad antifúngica de extractos etanólicos de dos morfotipos de Raphanus raphanistrum L. sobre tres hongos fitopatógenos. Bioagro: 27(1), 3-10 (2015) 
Sánchez, K. y Almaguer, M., Aeromicología y salud humana. Revista Cubana de Medicina Tropical: 66(3), 322-337 (2014)

Sanhueza, J.; Nieto, S. y Valenzuela, A., Ácido linoleico conjugado: un ácido graso con isomería trans potencialmente beneficioso. Revista Chilena de Nutrición: 29(2), (2002).

Santafé, G.G.; Guzmán, M.S. y Torres, O.L., Triterpenos Holostánicos con Actividad Antifúngica Obtenidos del Pepino de Mar Holothuria floridana, Recolectado en la Bahía de Cispatá, Córdoba-Colombia. Información Tecnológica: 25(2), 87-92 (2014)

Wang, Z. y otros 8 autores. Antifungal nortriterpene and triterpene glycosides from the sea cucumber Apostichopus japonicus Selenka. Food Chemistry: 132, 295-300 (2012)

Zamorano, P. y Leyte Morales, G., Cambios en la diversidad de equinodermos asociados al arrecife coralino en la Entrega, Oaxaca, México. Ciencia y Mar: 9(27), 19-28 (2005)

Zapata, K.; Cortes, F. y Rojano, B., Polifenoles y Actividad Antioxidante del Fruto de Guayaba Agria (Psidium araca). Información Tecnológica: 24(5), 103-112 (2013)

Zhao, Y. y otros 5 autores. Effects of potential probiotic Bacillus subtilis T13 on growth, immunity and disease resistance against Vibrio splendidus infection in juvenile sea cucumber Apostichopus japonicus. Fish \& Shellfish Immunology: 32, 750-755 (2012)

Zhou, D.Y. y otros 11 autores. Extraction and antioxidant property of polyhydroxylated naphthoquinone pigments from spines of purple sea urchin Strongylocentrotus nudus. Food Chemistry: 129, 1591-1597 (2011) 